\title{
Mathematical Modelling of Volatile Gas Using Lattice Boltzmann Method
}

\author{
Alok DHAUNDIYAL ${ }^{1 *}$, Suraj BHAN SINGH ${ }^{2}$ \\ ${ }^{1}$ Szent Istvan University, Doctoral School of Mechanical Engineering, Godollo, Hungary \\ ${ }^{2}$ Department of Mathematics, Statistics and Computer Science, Govind Ballabh Pant University of \\ Agriculture and Technology, Pantnagar, Uttarakhand, India
}

\begin{abstract}
This study investigates the behaviour of pyrolysis gas, generated by the thermal decomposing of biomass, in a pilot size reactor. The discreet mathematical model, Lattice Boltzmann, has adopted for mathematical simulation of flow of pyrolysis gas across a porous bed of biomass. The effect of permeability, pressure gradient, voidage of bed, density, temperature, and the dynamic viscosity on the mass flow rate of gas is examined by simulating the gas flow across the fixed bed of hardwood. The Darcy equation is used to estimate the flow rate of gas across the fixed bed of hardwood chips. The temperature in the reactor varies from $32{ }^{\circ} \mathrm{C}$ to $600{ }^{\circ} \mathrm{C}$. The reactor has an external diameter of $220 \mathrm{~mm}$ and the vertical height of $320 \mathrm{~mm}$. Rockwool insulation is used to prevent heat loss across the reactor. The external heating element of $2 \mathrm{kWe}$ was provided to trigger the pyrolysis reaction. The properties of the system have been recorded by the pressure and temperature sensors, which are retrofitted along the periphery of the reactor. The temperature sensors are located at $80 \mathrm{~mm}$ apart from each other; whereas the pressure sensor, placed at the bottom circumference of the reactor. The effect of input parameters on the flow properties of gas is also examined to add up the qualitative assessment of the system to biomass pyrolysis. The polytropic equation of gas is found to be $P V^{2.051}=C$, whereas the compressibility of gas varies from $0.0025-0.042 \mathrm{~m}^{2} \cdot \mathrm{N}^{-1}$.
\end{abstract}

Keywords - Darcy equation; flow properties; hardwood; Lattice Boltzmann method; numerical solution; modelling; pyrolysis gas.

\begin{tabular}{|lll|}
\hline \multicolumn{2}{l}{ Nomenclature } & $\mathrm{d}$ \\
$K$ & Permeability & $\mathrm{m} \cdot \mathrm{s}^{-1}$ \\
$\bar{v}_{S}$ & Superficial velocity & $\mathrm{Pa}$ \\
$G$ & Bulk modulus & - \\
$N$ & Polytropic index & $\mathrm{kg} \cdot \mathrm{m}^{-3}$ \\
$\rho_{\mathrm{s}}$ & Solid material density & $\mathrm{kg} \cdot \mathrm{m}^{-3}$ \\
$\rho_{\mathrm{b}}$ & Bulk density & - \\
$C$ & Sutherland constant & $\mathrm{N} \cdot \mathrm{s} \cdot \mathrm{m}^{-2}$ \\
$\mu$ & Dynamic viscosity & $\mathrm{Pa}$ \\
$P$ & Pressure & $\mathrm{m} \cdot \mathrm{s}^{-1}$ \\
\hline$c_{\mathrm{s}}$ & Velocity of sound in lattice & \\
\hline
\end{tabular}

* Corresponding author.

E-mail address: alok.dext@hotmail.com 


\begin{tabular}{|lll|}
\hline$\Phi$ & Voidage & - \\
$T$ & Temperature & $\mathrm{K}$ \\
$T_{0}$ & Reference temperature (STP) & $\mathrm{K}$ \\
$\mu_{0}$ & Viscosity at STP & $\mathrm{N} \cdot \mathrm{s} \cdot \mathrm{m}^{-2}$ \\
$\bar{V}$ & Average velocity from LBM & $\mathrm{m} \cdot \mathrm{s}^{-1}$ \\
$B$ & Width of reactor & $\mathrm{m}$ \\
$A_{\mathrm{c}, \mathrm{s}}$ & Cross sectional area of reactor & $\mathrm{m}$ \\
$v_{l}$ & Kinematic velocity & $\mathrm{m} \cdot \mathrm{s}^{-1}$ \\
$v_{x}$ & Velocity component along X-axis & $\mathrm{m} \cdot \mathrm{s}^{-1}$ \\
$v_{y}$ & Velocity component along Y-axis & $\mathrm{m} \cdot \mathrm{s}^{-1}$ \\
$\omega$ & Relaxation frequency & $\mathrm{s}^{-1}$ \\
$\tau$ & Relaxation time & $\mathrm{s}^{-1}$ \\
$v_{y, a}$ & Velocity along Y-axis (analytical solution) & $\mathrm{m} \cdot \mathrm{s}^{-1}$ \\
$v_{m}$ & Maximum velocity on the velocity profile (analytical) & $\mathrm{m} \cdot \mathrm{s}^{-1}$ \\
$Q$ & Volumetric rate of gas & $\mathrm{m} \cdot \mathrm{s}^{-1}$ \\
$\dot{m}$ & Mass flow rate of gas & $\mathrm{kg} \cdot \mathrm{s}^{-1}$ \\
\hline
\end{tabular}

\section{INTRODUCTION}

It is very important to know the dynamics of the gas flow inside a pyrolysis reactor since the major objective of any energy sector is to delimitate the flow problem of biomass. The design of the reactor is decided in such a manner that it would reduce the menial effort for running a power plant. In the biomass power plant, the behaviour of biomass and its flowing pattern decides the extent of the thermochemical conversion. A poorly managed power plant has a lower yield than that of a well-analysed power unit. The synergy of biomass characteristics, operating conditions and the design of the reactor decide the qualitative aspect of the power plant. If the attention is focused upon the flow behaviour of biomass and its conversion rate, the bridging and channelling effect of loose biomass cannot be overruled. The cumulative effect of bridging and channelling hampers the autocatalytic reactions; and thus, the residence time gets shorter, percentage of tar yield increases, and the fraction of residual mass becomes relatively high. The comparison of the superficial velocity with the velocity of fluid across the porous medium helps to understand the flow behaviour.

Some industries are taking preventive measures to shun the side effect of loose biomass. The procurement cost of the loose biomass is very low, but the problems associated with the flow pattern of biomass must be addressed efficiently. Some construction-related methods are being used for rice husk power plants such as modification of angle of repose of hopper, coupled-cone cylinder shell for feeding the biomass, the simultaneous removal of char and ash with the help of a stepper motor, using double cone section, and installation of a vibratory feeder to change to bulk density at the time of operation. Apart from the basic constructional design, some factors are to be examined. These factors are the operating conditions and their subsequent effect on the flow of producer gas. To simulate the behaviour of the gas, an interface is required, therefore the Lattice 
Boltzmann method (LBM) is adopted to understand the relationship between the fluid flow and the characteristic of biomass. But before doing that, the basic information related to the LBM must be known. The LBM is derived from the lattice gas automata (LGA) which gives information about the flow properties in terms of Boolean algebra. But owing to the statistical noise and lack of Galilean invariance, it has been phased out by the LBM. Also, instead of Boolean algebra, the ensemble averages of flow properties in the form of the density distribution function is used in the LBM. The LBM can be used for simulation for momentum, mass, and the energy transport processes. The LGA was one of useful computational fluid dynamics (CFD) [1]. But with time, the LBM becomes more prominent for the simulation of multiphase flow [2], [3]. Unlike the conventional CFD models which solve macroscopic equations, the LBM pivots on mesoscopic kinetic models. The phase segregation and interfacial dynamics, which are significant parts of multiphase flow and are difficult to tackle, can be solved easily with the LBM. The first LBM scheme for the multiphase problem had been propounded in 1991 [4]. A two-layer Couette/Poiseuille flow problem has been solved by the LBM and it has been found that the long waves on a viscosity interface can be unstable at any Reynold number [5]. Thereafter, numerous works have been focused on the effects of viscosity ratio, density ratio, and the height of interface [6]-[8]. Likewise, the method of Shan and Chen [9], the energy method of Swift et al. [10], and the method of He and co-workers [11]-[13]. The effect of viscosity ratio, surface tension, wettability and the capillary number has been examined in literature [14]-[17]. These are some of the methods which have been currently employed in solving the fluid-related problem.

The objective and significance of the current work are related to examining the effect of a porous medium, properties of the system and the flow properties on the production of gas through biomass pyrolysis. The magnitude and the qualitative analysis of mass flow rate through porous space provide information about mutual interaction between the biomass and the gas, thus the inclusion of the solid boundary is essential for predicting the behaviour of the gas flow past an obstruction. The Darcy equation for the porous medium is used to evaluate the effect of permeability of the solid medium, pressure gradient and dynamic viscosity of the gas on the mass flow rate.

\section{Material AND MethodS}

\subsection{Experimental Set-Up}

The tests are conducted on the hardwood (Acacia) chips (G50) in a small-scale reactor. The processed raw material is derived through shredding of wood. The thermodynamic properties of system (pressure and temperature) are measured by pressure and temperature sensors located along periphery of the reactor. The temperature sensors are kept at $80 \mathrm{~mm}$ from each other. The volumetric flow of gas is set to be $70 \mathrm{~mL} / \mathrm{s}$. The ' $\mathrm{K}$ ' type thermocouple is used for experimental purposes. The weight sensor is placed underneath the heating chamber which is separated from the rockwool insulation. The indirect heating is provided by a heating element of $2 \mathrm{kWe}$ (Hertz, Germany). Stainless steel material is used to manufacture the grate of $0.7 \mathrm{~mm}$ thickness, whereas the heat insulation is provided by rock wool. The insulation thickness of $50 \mathrm{~mm}$ is used to separate the core unit from the outer casing. The core of the heating chamber is made up of $1.5 \mathrm{~mm}$ welded carbon steel. The inner diameter of the reactor is $110 \mathrm{~mm}$, whereas the surface diameter of the heating chamber is $210 \mathrm{~mm}$. The pictorial diagram of the pyrolysis unit is illustrated in Fig. 1 . Elemental composition of the raw material is measured by the CHNS (Carbon, Hydrogen, Nitrogen and Sulphur) analyser (vario MACRO cube, Germany). The Halogen content in hardwood is determined using Infrared detector (IR), whereas the bomb calorimeter is employed for measuring the calorific value of hardwood. The chemical composition of hardwood (Acacia) 
is given in Table 1. The gas composition obtained by pyrolysis of hardwood is provided in Table 2 . The physical properties of hardwood are mentioned in Table 3.

TABLE 1. CHEMICAL COMPOSITION OF HARDWOOD CHIP

\begin{tabular}{llllllll}
\hline $\mathbf{C}, \boldsymbol{\%}$ & $\mathbf{H}, \boldsymbol{\%}$ & $\mathbf{N}, \boldsymbol{\%}$ & $\mathbf{O}, \boldsymbol{\%}$ & $\mathbf{S ,} \%$ & Ash, \% & $\mathbf{C l}, \boldsymbol{\%}$ & Net calorific value, $\mathbf{M J} / \mathbf{k g}$ \\
50.03 & 5.849 & 0.075 & 42.947 & 0.061 & 1.037 & 0.02 & 18.70 \\
\hline
\end{tabular}

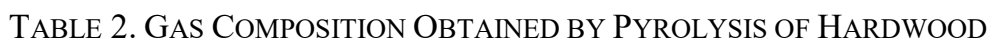

\begin{tabular}{lllll}
\hline $\mathbf{C O}$, & $\mathbf{H}_{2}$, & $\mathbf{C H}_{4}$, & $\mathrm{CO}_{2}$, & $\mathbf{N}_{2}$, \\
$\%$ & $\%$ & $\%$ & $\%$ & $\%$ \\
14.3 & 19.9 & 1.8 & 12.2 & 51.8 \\
\hline
\end{tabular}

TABLE 3. PhysiCAL Properties OF HARDWOOd CHIPS

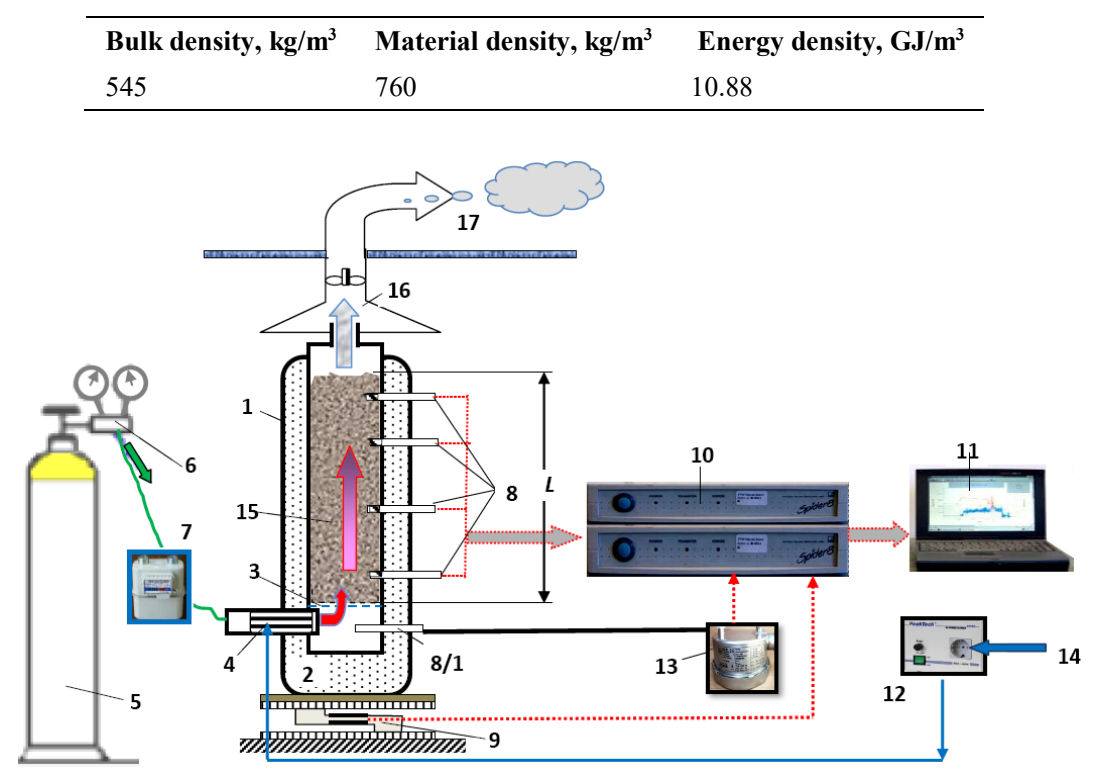

Fig. 1. Schematic diagram of the pyrolysis unit ( 1 - main body, 2 - insulator, 3 - reactor, 4 - heating element, $5-\mathrm{N}_{2}$ cylinder, 6 - pressure valve, 7 - gas meter, 8 - temperature sensors, $8 / 1$ - pressure probe, 9 - strain gauge, 10 - data logger, 11 - computer, 12 - heating unit, 13 - pressure sensor for reactor, 14 - electrical relay, 15 - feedstock, 16 - chimney, 17 - exhaust outlet).

\subsection{Lattice Boltzmann Method for Porous Medium}

The Lattice Boltzmann method is a numerical technique to solve the fluid-related problem. The Boltzmann Eq. (1), in the discrete form, is used to describe the dynamics of a gas from a mesoscopic viewpoint. A gas particle moving with velocity, $v$, collides with a certain probability and transfers the momentum to its neighbourhood particle. In an ideal situation, the total momentum and energy are conserved whilst successive collisions occur. The Boltzmann equation expresses how the probability function, $f(z, t)$, associated with the location of a particle having velocity, $v$, evolves with time, $t$. 


$$
v \cdot \nabla_{z} f+F \cdot \nabla_{p} f+\frac{\mathrm{d} f}{\mathrm{~d} t}=\Omega(f(z, t)),
$$

where

$F \quad$ external body force;

$\nabla_{z, p}$ gradient in position and momentum space;

$\Omega \quad$ collision operator.

Bhatnagar, Gross and Krook [18] propounded the so-called BGK dynamics, wherein the collision operator $\Omega$ is opted in such a manner that the Eq. (1) can be correlated with the relaxation time of a dynamic system (Eq. (2)).

$$
\Omega[f(z, t)]=-\frac{\left[f(z, t)-f^{E q}(z, t)\right]}{\tau},
$$

where $\tau$ is the relaxation time, and the position of node on a two-dimensional lattice is denoted by $z \equiv(x, y)$ and time by $t$.

In Eq. (2), the equilibrium distribution function $f^{E q}(z, t)$, for a thermal model depends on the local density $\rho(z, t)$ and the velocity field $v(z, t)$ (Eq. (3) and Eq. (4)). The Lattice Boltzmann method [19] assists to discretise the probability density function $f(z, t)$ in space and time. The discretisation and the analytic solution for the equilibrium distribution is carried out according to the lattice type. In the following analysis, a D2Q9 lattice (Fig. 2.) is being used for simulation of volatile gas. Each lattice site has a unique value which is denoted by lattice vector or velocity, $c_{i}$.

The flow properties of lattice fluid can be defined for index $k$ as:

$$
\begin{gathered}
\rho(z, t)=\sum_{n=0}^{n=k-1} c_{i} f(z, t), \\
\rho(z, t) v(z, t)=\sum_{n=0}^{n=k-1} c_{i} f(z, t) .
\end{gathered}
$$

The equilibrium value is obtained by discretising the Boltzmann distribution. The various forms of different order of $f_{i, e q}(\rho, t)$ have been reported in literature, but the most common form in term of lattice velocity has been used [20]-[24]. The equilibrium distribution function is given by:

$$
f_{i, e g}(\rho, t)=w_{i} \rho\left[1+\frac{\left(c_{i} v\right)}{c_{s}^{2}}+\frac{\left(c_{i} v\right)^{2}}{2 c_{s}^{4}}-\frac{\left(v^{2}\right)}{2 c_{s}^{2}}\right]
$$

with the weight coefficient

$$
w_{i}=\left\{\begin{array}{lc}
\frac{4}{9}, & i=0 \\
\frac{1}{9}, & i=1,2,3,4 \\
\frac{1}{36}, & i=5,6,7,8
\end{array}\right.
$$




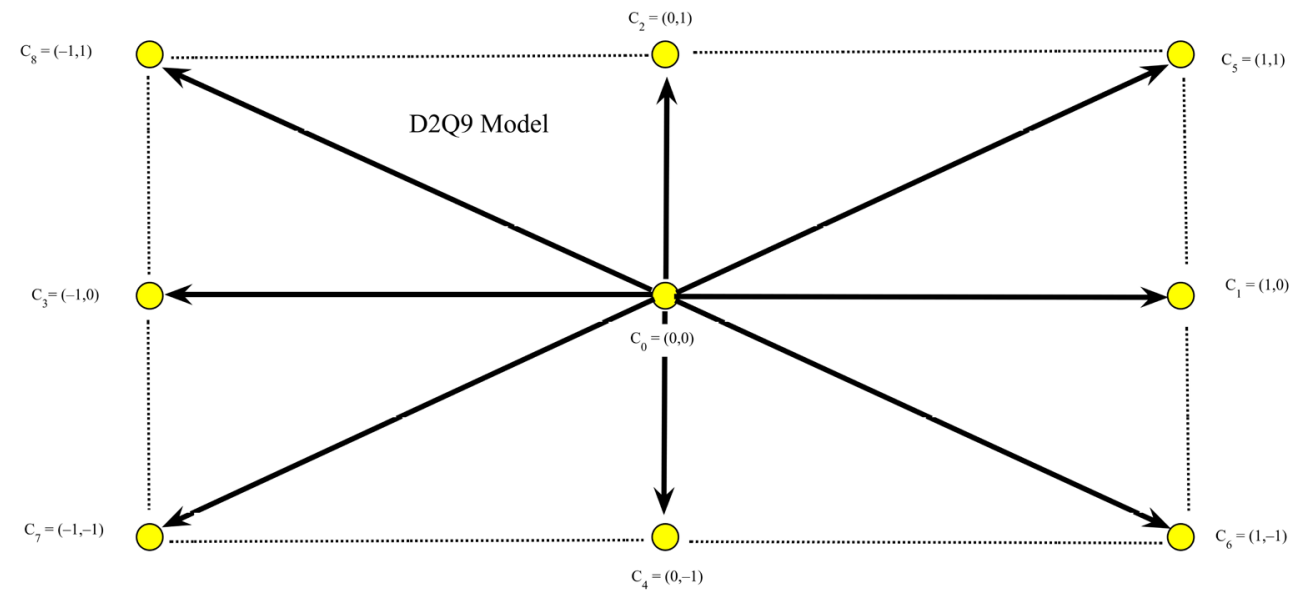

Fig. 2. The geometry of D2Q9 lattice with the lattice velocity $c_{i}$.

The set of velocities, $C$, for the 2D model, D2Q9, is

$$
C=\left[\begin{array}{rrllllllr}
0 & 1 & 0 & -1 & 0 & 1 & -1 & -1 & 1 \\
0 & 0 & 1 & 0 & -1 & 1 & 1 & -1 & -1
\end{array}\right]
$$

The boundary conditions for the given fluid problem are derived by using the set of equations, which are obtained by incorporating Eq. (3) and Eq. (4).

$$
\begin{aligned}
& \rho v_{x}=\left(f_{2}+f_{6}+f_{9}\right)-\left(f_{4}+f_{7}+f_{8}\right) \\
& \rho v_{y}=\left(f_{3}+f_{6}+f_{7}\right)-\left(f_{5}+f_{8}+f_{9}\right)
\end{aligned}
$$

Using the continuity equation, $\frac{\partial \rho}{\partial t}+\nabla \cdot(\rho v)=0$, the Neumann boundary condition can be imposed on the boundary where one of the velocity components is fixed and density is invariant of time.

By solving Eq. (3) and Eq. (4) for an initial value of density $\rho_{0}$ at boundary and a fix value of $v_{x}$, we get

$$
v_{y}=1-\frac{\left[\left(f_{1}+f_{2}+2\left(f_{5}+f_{8}+f_{9}\right)\right]\right.}{\rho_{0}}
$$

or

$$
\rho=\frac{\left[\left(f_{1}+f_{2}+2\left(f_{5}+f_{8}+f_{9}\right)\right]\right.}{1-v_{y}} .
$$

The function $f_{i}$ with the weight factor ' 2 ' represents components pointing out of the system, whereas those which have unit weight are all-in line components. On the other hand, the terms which have not appeared are undetermined after the streaming step. 
The Eq. (9) can also be generalised for 3D system as well, whereas the density of the system is known by Eq. (10) which is Dirichlet or flux boundary condition. It is also clear from Eq. (10) that the undetermined population do not contribute to the evaluation of density at a particular location and time. The population that participates in the computational process can be estimated by applying the bouncing back condition on the boundary [25], thus the non-equilibrium part of collision step can be estimated by Eq. (11) as

$$
f_{i-1}^{\prime}=f_{i-1}-f_{i-1}^{E q}=f_{i}^{\prime}=f_{i}-f_{i}^{E q} .
$$

Thus,

$$
\begin{aligned}
& f_{i-1}=f_{i}+\rho\left(w_{i-1}-w_{i}\right)+ \\
& +\rho\left[w_{i-1}\left[\frac{\left(c_{i-1} v_{y}\right)}{c_{s}^{2}}+\frac{\left(c_{i-1} v_{y}\right)^{2}}{2 c_{s}^{4}}-\frac{\left(v_{y}^{2}\right)}{2 c_{s}^{2}}\right]-w_{i}\left[\frac{\left(c_{i} v_{y}\right)}{c_{s}^{2}}+\frac{\left(c_{i} v_{y}\right)^{2}}{2 c_{s}^{4}}-\frac{\left(v_{y}^{2}\right)}{2 c_{s}^{2}}\right]\right] .
\end{aligned}
$$

The relaxation frequency, $\omega$, can be determined by using the lattice kinematic viscosity of the gas, $v_{l}$ by

$$
w=\frac{1}{\left(v_{l} \cdot c_{\mathrm{s}}^{2}+0.5\right)} .
$$

The simulation task has been performed on MATLAB 2015b. The coding is based on the solid boundary generation with the help of voidage, which is obtained experimentally. The experimentally measured value of average velocity considers as the reference point about which the algorithm has been converged. The initial velocity of gas at $t=0$ is considered to be the velocity of scavenging gas, nitrogen. The simulation process is carried out by using the experimental values of fluid properties, whereas the lattice velocity of sound is estimated with the help of the polytropic law $P V^{\mathrm{n}}=C$. The numerical solution keeps comparing with its analytical counterpart until the solution converges. The tolerance range of $10^{-8}$ is assumed to be a minimum threshold to terminate the iterative loop. The biomass boundaries are divided into two parts: wet location and dry location, thus the seepage velocity is obtained by using a logical function. The local equilibrium function $f^{(0)}$ is calculated, with the given initial density $\rho(z, t)$ and momentum, thereafter set $f_{i}^{(1)}=f_{i}^{(0)}$. Use the iterative concept and propagate it to the next neighbour (except for the distribution of stationary particle with $c_{0}=0$ ). With the help of new distribution function, evaluate the new density (Eq. (3)) and momentum (Eq. (4)). After estimating the new local equilibrium distribution, the propagate step has been repeated.

The permeability of a porous system is defined as

$$
K=\bar{V} \cdot\left(\frac{\varphi}{\frac{\partial P}{\partial x}}\right) \cdot v_{l} .
$$

The $\varphi$ denotes the voidage of bed, $\bar{V}$ is the average velocity of gas, and $\frac{\partial P}{\partial x}$ is the pressure gradient. 


$$
\varphi=1-\frac{\rho_{\mathrm{b}}}{\rho_{\mathrm{s}}}
$$

The $\rho_{\mathrm{b}}$ represents the bulk density of biomass, and $\rho_{\mathrm{s}}$ is the material density.

The Darcy equation for measuring the volumetric rate across the porous medium is defined as

$$
Q=-\frac{K A_{\mathrm{c}, \mathrm{s}}}{\mu} \cdot \frac{\partial P}{\partial x},
$$

where

$Q \quad$ volumetric discharge of the gas;

$A_{\text {c.s }} \quad$ cross-section of the reactor;

$\mu \quad$ dynamic viscosity;

$\frac{\partial P}{\partial x}$ pressure gradient along the direction of flow.

The mass flow rate of gas is estimated by Eq. (17)

$$
\dot{m}=\rho(x, t) \cdot Q .
$$

The analytical solution of the velocity profile is given by

$$
v_{y, a}=v_{m}\left(1-4\left(\frac{x}{B}\right)^{2}\right),
$$

where

$v_{y, a} \quad$ velocity obtained by the analytical solution;

$v_{m} \quad$ maximum velocity on the velocity profile;

$B \quad$ width of the reactor;

$x \quad$ general position along the radial direction.

$$
v_{m}=-\frac{1}{16 \mu} \frac{\partial P}{\partial x} B^{2}
$$

The gas viscosity is calculated by using Sutherland's formula [26], valid for input temperature range of $0 \leq T(K) \leq 811$

$$
\mu=\mu_{0}\left(\frac{a}{b}\right)\left(\frac{T}{T_{0}}\right)^{\left(\frac{3}{2}\right)},
$$

where $a=0.555 T_{0}+C$ and $b=0.555 T_{0}+C$. 
TABLE 4. PARAMETRIC INFORMATION FOR THE LB SIMULATION

\begin{tabular}{lllllll}
\hline $\boldsymbol{\mu}, \mathbf{N} \cdot \mathbf{s} \cdot \mathbf{m}^{-2}$ & $\boldsymbol{\rho}, \mathbf{k g} \cdot \mathbf{m}^{-3}$ & $\boldsymbol{G}, \mathbf{P a}$ & $\boldsymbol{n}$ & $\frac{\partial \boldsymbol{P}}{\partial x}, \mathbf{P a} \cdot \mathbf{m}^{-\mathbf{1}}$ & $\boldsymbol{C}$ & $\boldsymbol{\mu}_{0,} \mathbf{N} \cdot \mathbf{s} \cdot \mathbf{m}^{-\mathbf{2}}(\mathbf{S T P})$ \\
\hline 0.0000567 & 2.12413 & 116.098 & 2.0051 & 177.369 & 121 & 0.0000144 \\
\hline
\end{tabular}

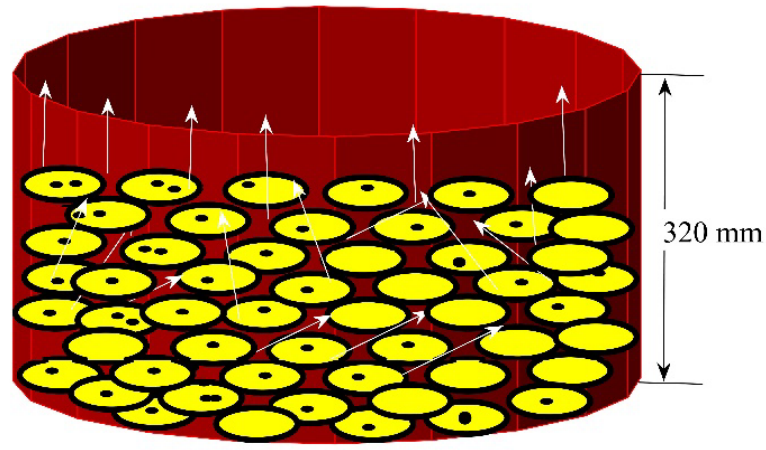

Fig. 3. Graphical illustration of the system.

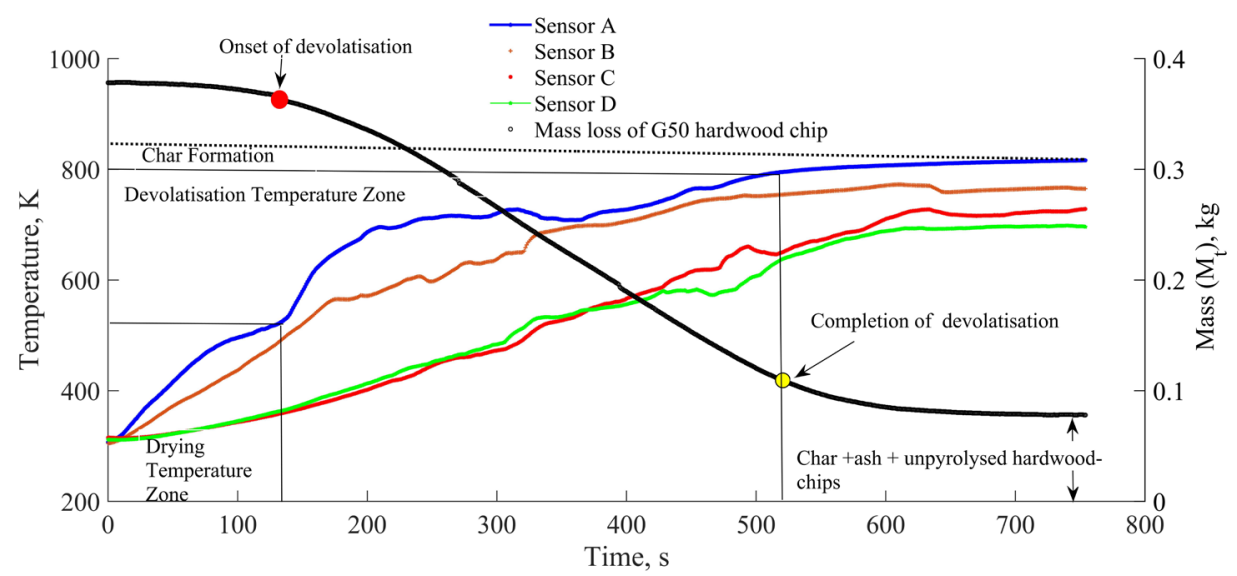

Fig. 4. Thermogravimetric variation of G50 Hardwood chip and the thermal history of the reactor with respect to time. 


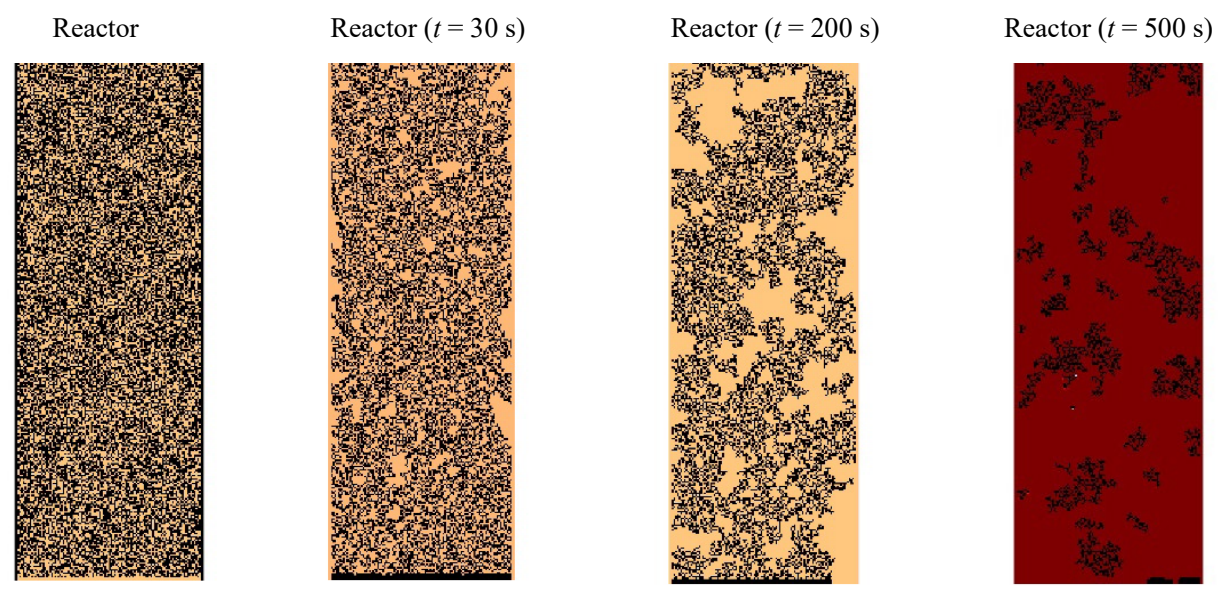

Fig. 5. Visualisation of biomass at different time frame.

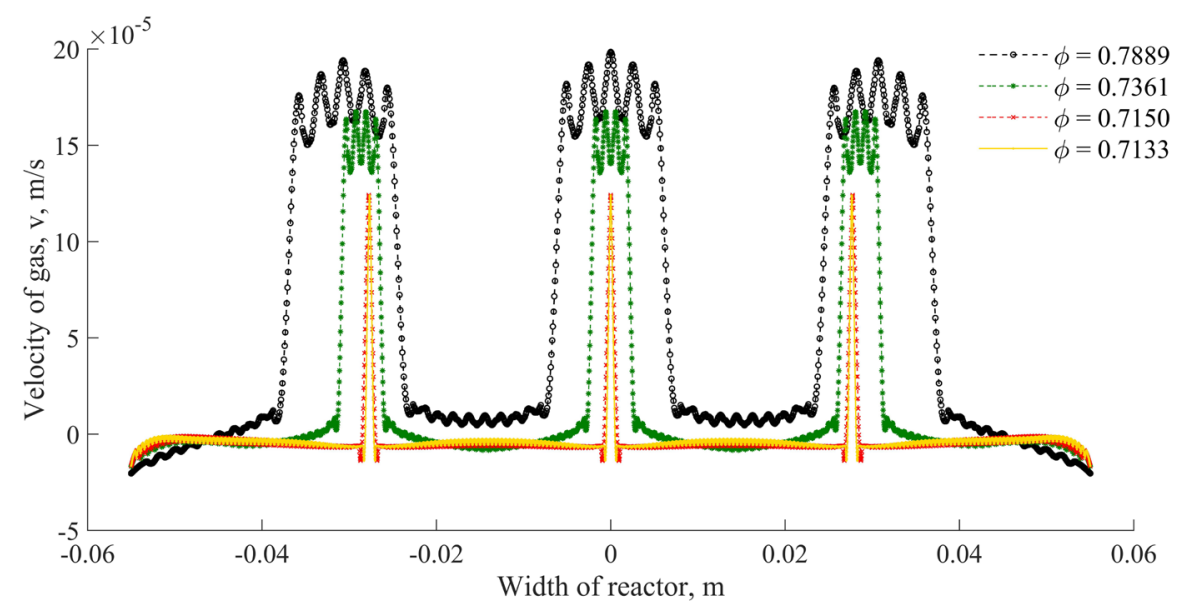

Fig. 6. The effect of voidage of the fixed bed $(\varphi)$ on the velocity profile of gas at constant pressure gradient, lattice speed (cs) and dynamic viscosity $(\mu)$. 


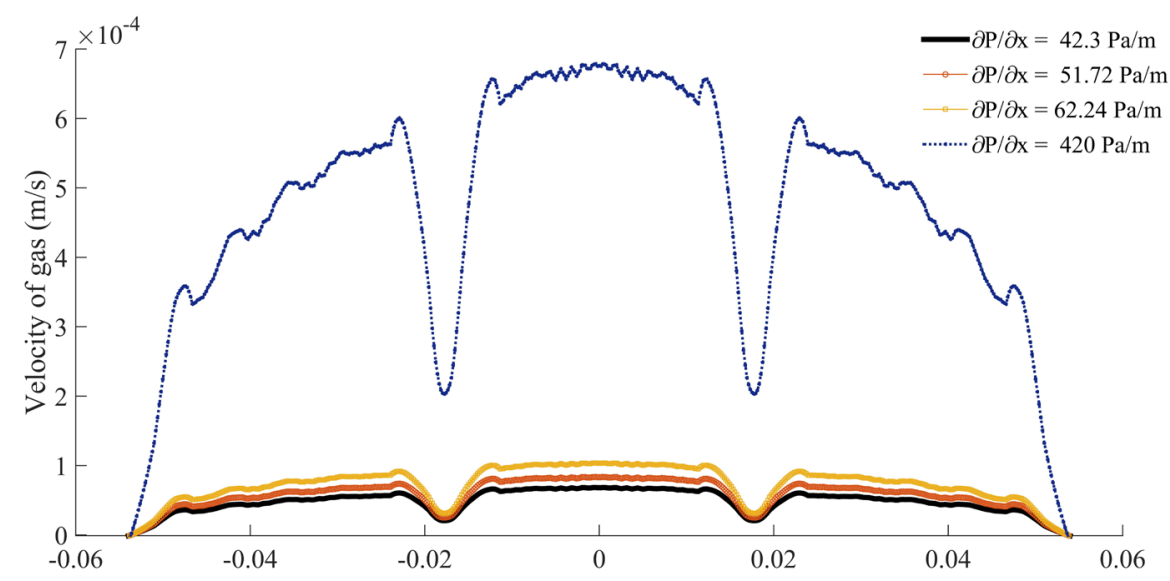

Fig. 7. The effect of pressure gradient on the velocity profile of gas at constant dynamic viscosity $(\mu)$ and lattice speed of sound (cs).

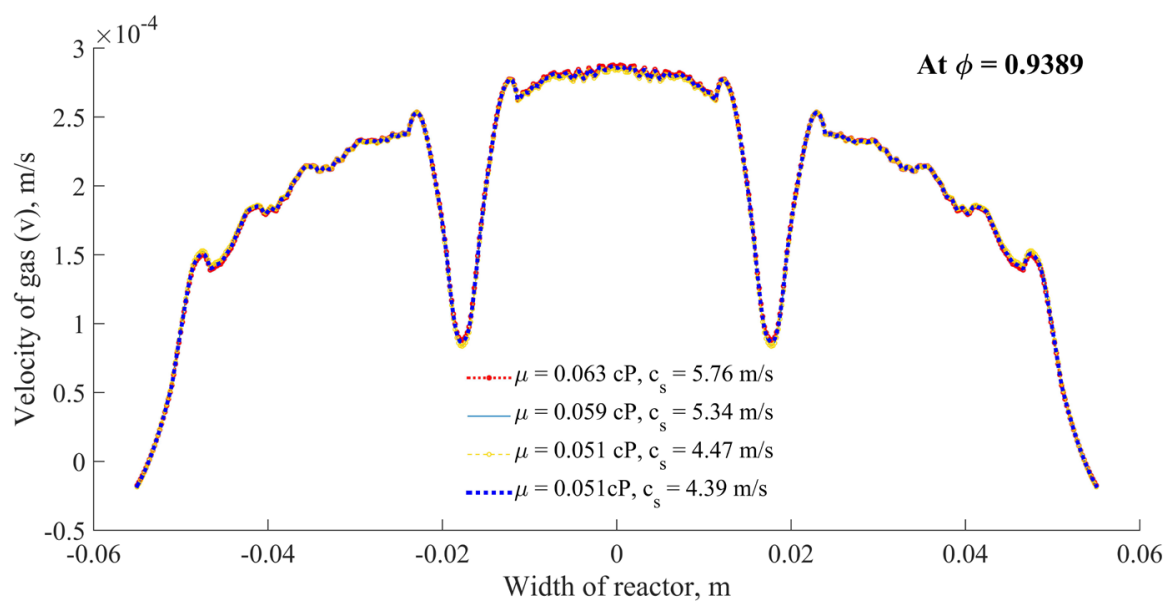

Fig. 8. Variation of velocity of volatile gas $(v)$ with respect to dynamic viscosity $(\mu)$ and lattice speed of sound $\left(c_{\mathrm{s}}\right)$ at constant voidage $(\varphi)$. 


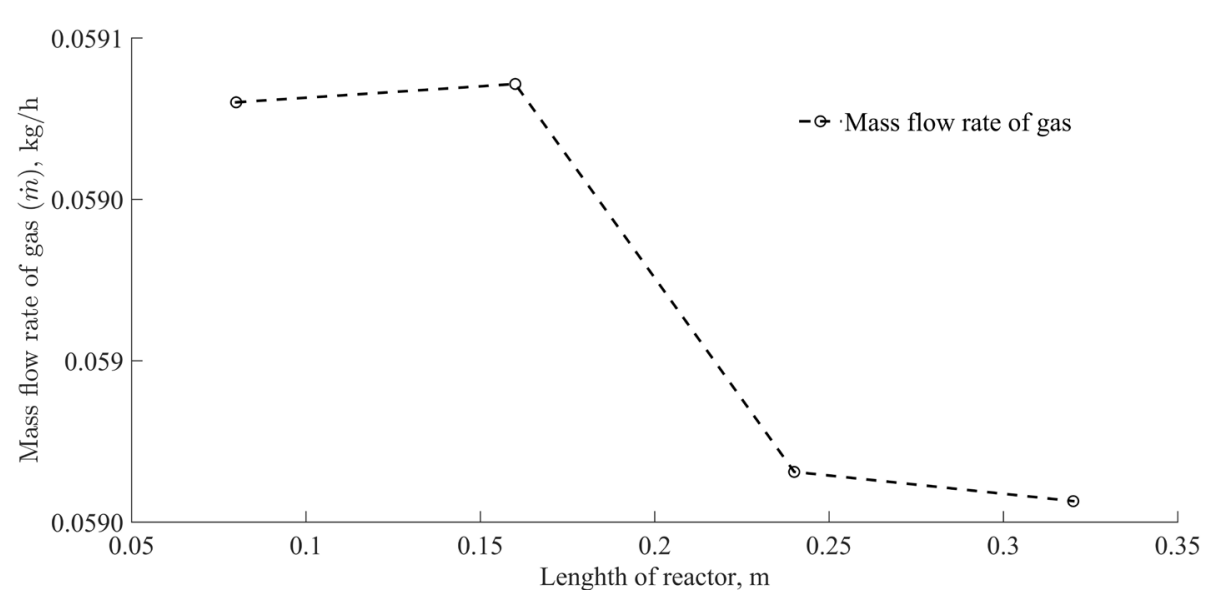

Fig. 9. The effect of dynamic viscosity $(\mu)$ on the mass flow rate of volatile gas ( $m$ ) along the reactor at constant pressure gradient.

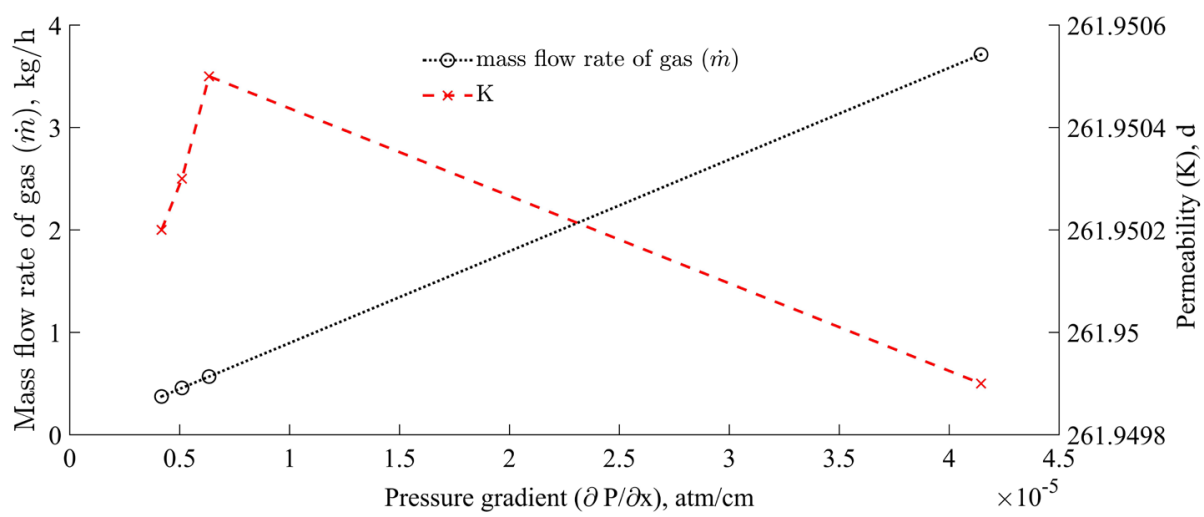

Fig. 10. The effect of pressure gradient $\left(\frac{\partial P}{\partial x}\right)$ on the mass flow rate of volatile gas. 


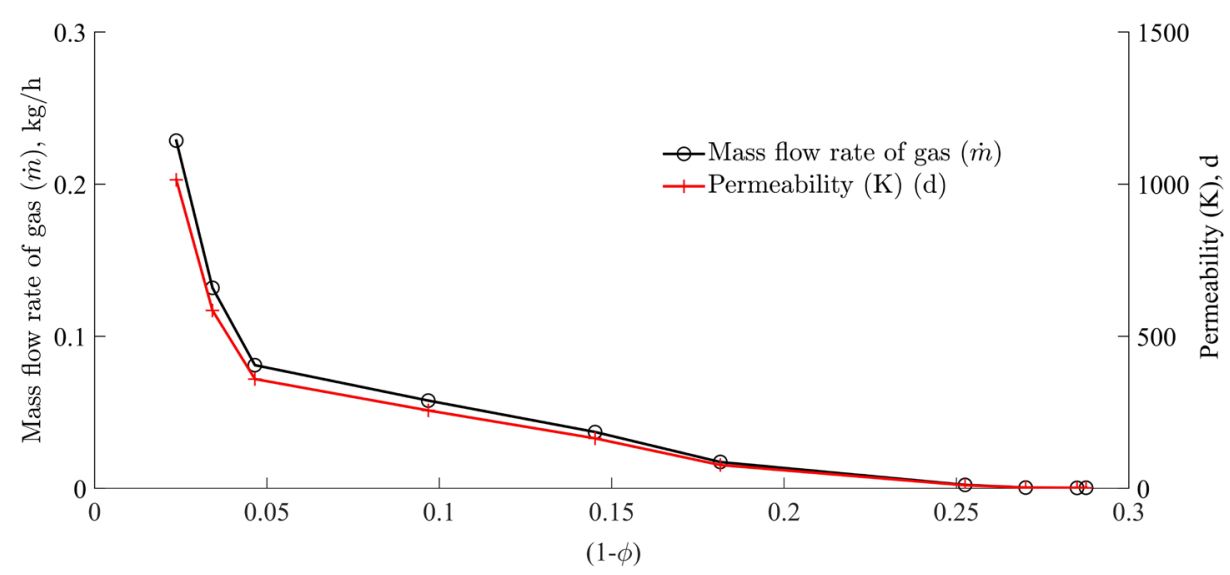

Fig. 11. Variation of mass flow rate of volatile gas $(m)$ and permeability $(K)$ with respect to voidage $(\varphi)$.

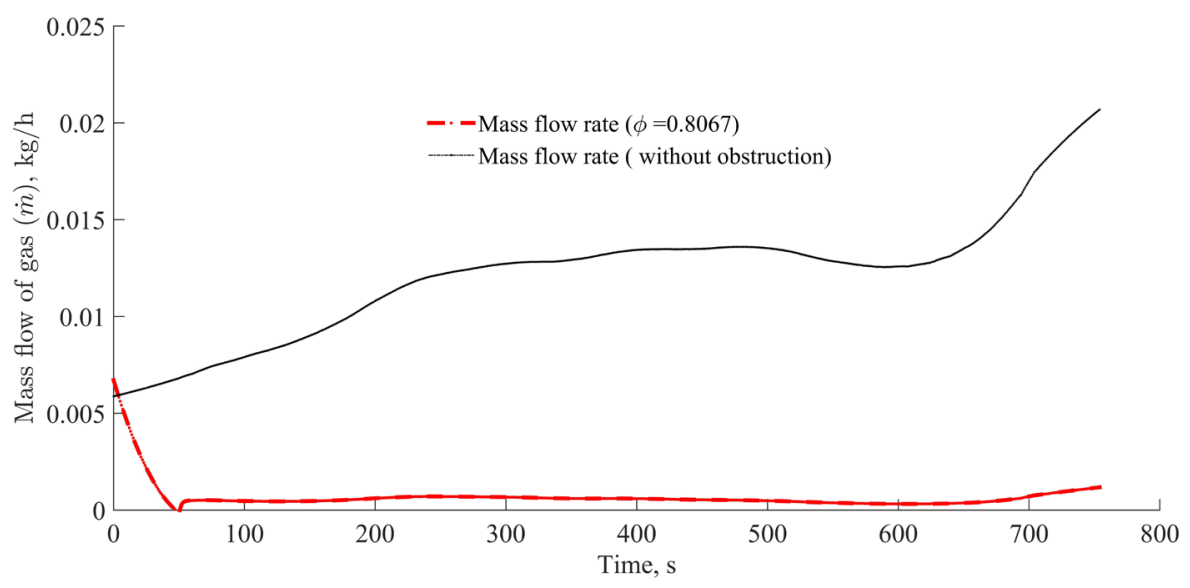

Fig. 12. Comparative illustration of flow medium (varying density of gas). 


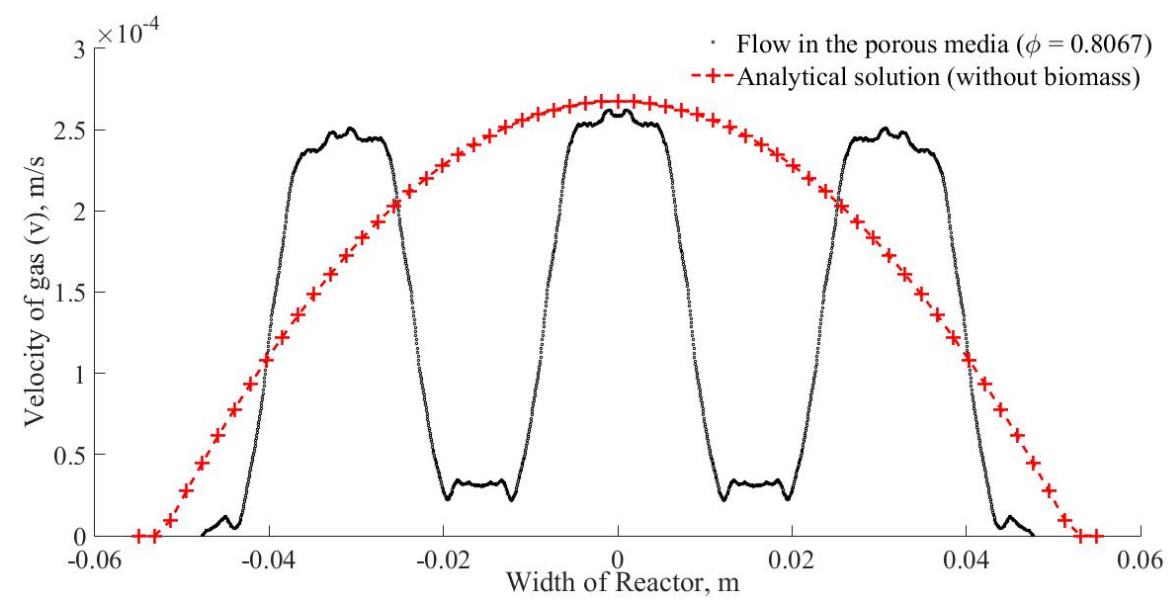

Fig. 13. Comparison of analytical solution with the LBM method.

\section{RESUlTS AND DisCUSSION}

The flow behaviour of pyrolysis gas generated during thermal degradation of G50 Hardwood across the porous medium is predicted with the help of the Lattice Boltzmann model. The D2Q9 lattice is used to determine the velocity of gas through the fixed bed having a voidage $\varphi$. The graphical representation of the system is illustrated in Fig. 3. The significance of analysis can be determined by the fact that the problem faced during operation of gasifier or pyrolysis reactor can be examined through detailed analysis of operational parameters (pressure gradient, temperature, flow rate across the porous medium, voidage, dynamic viscosity and density). Involvement of temperature in the study is incorporated with the help of lattice speed of sound which is a function of temperature. The temperature has been monitored at every $80 \mathrm{~mm}$ by the temperature sensors located along the reactor. The thermogravimetric variation and thermal history of the heating chamber is illustrated in Fig. 4. The drying of hardwood chip takes place in the range of temperature from $306 \mathrm{~K}$ to $500 \mathrm{~K}$. The onset of devolatisation initiates at $500 \mathrm{~K}$ and it gets completed at $800 \mathrm{~K}$. After the completion of devolatisation, the char formation stage begins, and it comprises char, unpyrolysed hardwood, and ash content. It is not necessary that the whole biomass gets processed; thus, a small fraction of biomass does not undergo the thermal degradation, which is collected from the grid material once the process is called off. It can be noticed, as illustrated in Fig. 4, that the temperature of heating chamber is decreasing as the gas is flowing up and, in this way, the characteristic of the flow properties also gets changed.

Now, the flow through porous medium has its own significance since it is essential to see the effect of medium thwarting the passage of the gas. The gas properties also play a crucial role in determining the gas production by a power plant. These effects are graphically represented by varying the flow properties of a system. The effect of the increasing voidage at different time frames can be observed in Fig. 5. The effect of voidage on the numerical solution is shown in Fig. 6 . It is seen that the increasing voidage makes the gas expand more rapidly and it reduces the momentum thickness. It is observed that velocity of gas increases with the increasing voidage of the fixed bed. The small fluctuation in velocity happens due to the variation of permeability of medium with voidage and the shear stress provided by the solid boundary of biomass channel and the biomass itself. The amplitude and attribute of velocity profile get changed with the decreasing 
voidage. The concept of velocity profile variation may also be cleared with the varying viscosity of gas and simultaneous erosion of biomass boundary. The channelling is mainly an undesirable route taken by the gas through voids space. This premature escaping of gas leads to an ineffective thermochemical conversion. If the superficial velocity across the biomass increases, it will increase the residual weight of biomass, which may cause bridging problem for the incoming feedstock. Therefore, the gas must properly pyrolysis the biomass before it exits. Both pressure and temperature increase the viscosity of gas. The effect of pressure gradient on the velocity profile is illustrated in Fig. 7. The shear strain rate is more predominant as the pressure gradient nearly increases by $7 \%$. The shear thickening of gas is relatively high at higher pressure gradient than that of lower pressure gradient. It is significant to know mutual interaction between viscosity and the pressure gradient as both the factors affect the volumetric production rate of gas. The increase in the pressure gradient will increase the volumetric rate provided the viscosity of gas does not change with time. The factors, the pressure and the temperature, have a similar effect on the viscosity of the gas, therefore the residence time of gas can only be increased if the effect of pressure and temperature supersedes the effect of pressure gradient at the base of the reactor. In this manner, due to inertial effect, the biomass will collapse and allow the fresh intake of feedstock. The qualitative aspect of biomass conversion will be materialised once the biomass gets eaten away effectively with time and temperature [27]-[29]. The sudden reduction in the shear strain rate at surface of biomass is due to the reduction of pressure as the temperature of the centre of biomass reaches the furnace temperature. This process gets accelerated due to structural failure. Due to this internal fracture, permeability and local porosity gets affected and the gas flow rate is altered. These cracks usually happen due to moisture content in a fuel particle and decreasing pressure inside the fuel particle. The shear thickening of gas across the fixed bed is also asserted by Fig. 8 . The effect of viscosity on the velocity of the gas is shown in Fig. 8. The attribute of curves has not changed, but the amplitude is marginally increased. The increasing viscosity and lattice speed of sound increases the velocity of gas flow across the porous medium. The shearing strain rate is also marginally elevated at constant pressure gradient. The variation of mass flow rate of gas with varying viscosity along the length of reactor is shown in Fig. 9. It can be seen that permeability surpassed the viscosity of gas. Despite, at the base of the reactor, the gas viscosity is higher than that of the other sections of chamber and it is also inversely related to the volumetric flow rate of gas (Eq. (16)). Permeability variation with temperature and void is more susceptible than viscosity in itself, which can be much better understood by Fig. 11. However, the variation in mass flow rate is $0.16 \%$ which is quite negligible. The variation of permeability $(K)$ and mass flow rate of gas is shown in Fig. 10. The mass flow rate of gas outrightly increases with elevating pressure gradient. On the other hand, for the variation of less than $1 \%$ in the pressure gradient, the permeability will increase; but beyond the $1 \%$, it has decreasing trend with the pressure gradient. The relative merit of pressure gradient over permeability is very high for estimation of mass flow rate of gas across the porous medium. The influence of voidage $(\varphi)$ on the mass flow rate of gas and the permeability is illustrated in Fig. 11. It can be seen that the permeability is increasing exponentially with $\varphi$ voidage, whereas the mass flow rate of gas has followed the similar trend with some greater extent. The increasing voidage or the decreasing bulk density of biomass accelerates the flow rate of gas and permeability of fluid across the fixed bed. So, mass flow rate at the onset of pyrolysis will be controlled if the bed is densely packed, and thus, it affects prematurely escaping of the gas across the channel. For a much better understanding, a comparative plot is drawn (Fig. 12), and it is observed that the mass flow rate based on the superficial velocity is relatively high to the mass flow rate calculated across the porous medium. In the beginning, the mass flow rate decreases drastically, and after some time, it becomes nearly constant with time. The comparatively plot between the analytical solution and the LBM is shown 
in Fig. 13. The effect of porous boundaries can be noticed from the drawn plot. The shear effect offered by the surface of biomass causes the velocity gradient to relatively high to the laminar flow (in the absence of solid boundary of fuel).

A point to be noted that the small fluctuation arises due to slip velocity, i.e. difference of velocities of gas and buoyed up particles. The pressure drops across the bed elevated as the gas flow rate across the interstitial gaps between the particles increases. A point is eventually reached when the pressure drop across the bed becomes equal to the weight of the biomass per unit crosssectional area of the bed. The velocity at this critical point is called the minimum fluidisation velocity, at which the bed is called incipiently fluidised. With further increase in gas velocity, the particles get buoyed up due to the drag force, which is greater than the weight of the particle itself, created by the gas and imparts a turbulent eddy like motion. This leads to the particle mixing and soon, the equilibrium between the gas and particle is attained. This temporal displacement of particle before reaching the transport disengagement height (TDH) falls back to the bed due to gravity. The freeboard formation due to wake of gas bubble is transformed into the entrainment stage when the superficial velocity of gas becomes equal to the terminal velocity of entrained particles. This fluidisation regime between minimum fluidisation velocity and terminal velocity is very essential from the operation point of view of a reactor. The attrition of the higher densities is carried out by the generated thermal stresses and the fluidisation. The superficial velocity beyond the terminal velocity may lead to slugging in the smaller cross-sectional reactor and makes the bed more stable. The bridging and channelling, and the entrainment for the loose biomass can be encountered if the superficial velocity of the gas operates within the fluidisation regime. At the onset of pyrolysis conversion, the voidage does not immediately decrease until the gas velocity unlocks the particle pattern by causing an extra pressure to drop at the incipient fluidisation. Once the particle is escaped from the pattern, the pressure drops fall back to the value sufficient enough to support the weight of particle of the bed.

\section{Conclusions}

The LBM method is used to simulate the behaviour of the gas across the fixed bed regime. Geometry of a small-scale reactor is used to predict the behaviour of the mass flow rate of gas with respect to the operational parameters. Darcy equation for the porous medium is used to determine the flow rate of the gas. The input parameters are obtained by the LB simulation. The relative effect of permeability over the dynamic viscosity on the mass flow of gas is high, and the flow pattern is governed by the pressure gradient applied across the reactor. The pressure gradient and temperature played a very crucial role to control volumetric rate of gas. The dynamic viscosity marginally affects the velocity of gas. The effect of voidage on the shear strain rate is very high, and thus, it also influences the mass flow rate of gas. The flow of gas along the reactor decreases with the decreasing dynamic viscosity. This is due to the fact the permeability has shown the exponential behaviour as the bulk density of biomass decreases with time. The variation of voidage depends on the pressure drop and the pressure drop varies linearly with the superficial velocity. The decreasing bulk density of biomass increases the gas flow rate across the bed (Fig. 11). The gas flow rate remains nearly constant with time at the constant voidage, whereas the overall increase has been observed when the gas flow across the channel without solid boundaries of biomass. The fast fluidisation of the bed can be more promising if it is applied to the loose biomass since the solid-gas mixing is the main priority to maximise the thermochemical conversion of the biomass. The disengagement and effective degree of solid refluxing may maintain the minimum level of temperature uniformity in the reactor if the elutriated particles 
(elutriated by the fluidising gas above the terminal velocity) are recovered and returned back to the furnace.

The LBM has found to be very successful for small pilot size pyrolysis units. However, the homogenous system can be much better predicted with Navier Stoke's equations. The solid biomass retarded the flow rate of gas and thus permeability of gas across is affected, however the channelling of gas is significantly reduced. Thus, more uniform thermal decomposition can be obtained for denser biomass.

\section{REFERENCES}

[1] Frisch U., Hasslacher, B., Pomeau, Y. Lattice-Gas Automata for the Navier-Stokes Equation. Physical Review Letters 1986:56(14):1505-1508. https://doi.org/10.1103/PhysRevLett.56.1505

[2] Rothman, D. H., Zaleski S. Lattice-gas models of phase separation: Interfaces, phase transitions, and multiphase flow. Reviews of Modern Physics 1994:66(4):1417-1479. https://doi.org/10.1103/RevModPhys.66.1417

[3] Chen S., Doolen G. D. Lattice Boltzmann Method for Fluid Flows. Annual Review of Fluid Mechanics 2002:30(1):329 364. https://doi.rg/10.1146/annurev.fluid.30.1.329

[4] Gunstensen A. K., et al. Lattice Boltzmann model of immiscible fluids. Physical Review A 1991:43(8):4320-4327. https://doi.org/10.1103/PhysRevA.43.4320

[5] Yih C. S. Instability due to viscosity stratification. Journal of Fluid Mechanics 1967:27(2):337-352. https://doi.org/10.1017/S0022112067000357

[6] Yiantsios S. G., Higgins, B. G. Linear stability of plane Poiseuille flow of two superposed fluids. Physics of Fluids 1988:31:3225-3238. https://doi.org/10.1063/1.866933

[7] Boyd W. G. C. Shear-flow in stability at the interface between two viscous fluids. Journal of Fluid Mechanics 1983:128:507-528. https://doi.org/10.1017/S0022112083000580

[8] South M. J., Hooper A. P. Linear growth in two-fluid plane Poiseuille flow. Journal of Fluid Mechanics 1999:381:121139. https://doi.org/10.1017/S0022112098003577

[9] Shan X., Chen H. Lattice Boltzmann model for simulating flows with multiple phases and components. Physical Review E 1993:47(3):1815-1819. https://doi.org/10.1103/PhysRevE.47.1815

[10] Swift M. R., Osborn W. R., Yeomans J. M. Lattice Boltzmann simulation of nonideal fluids. Physical Review Letters 1995:75(5):830-833. https://doi.org/10.1103/PhysRevLett.75.830

[11] He X., et al. On the three-dimensional Rayleigh-Taylor instability. Physics of Fluids 1999:11(5):1143-1152. https://doi.org/10.1063/1.869984

[12] Wang Y., et al. Multiphase lattice Boltzmann flux solver for incompressible multiphase flows with large density ratio. Journal of Computational Physics 2015:280:404-423. https://doiorg/10.1016/j.jcp.2014.09.035

[13] Zhang R., He X., Chen S. Interface and surface tension in incompressible lattice Boltzmann multiphase model. Computer Physics Communications 2000:129(1-3):121-130. https://doi.org/10.1016/S0010-4655(00)00099-0

[14] Chin J., Boek E. S., Coveney P. V. Lattice Boltzmann simulation of the flow of binary immiscible fluids with different viscosities using the Shan-Chen microscopic interaction model. Philosophical Transactions of the Royal Society A: Mathematical, Physical and Engineering Sciences 2002:547-558. https://doi.org/10.1098/rsta.2001.0953

[15] Grosfils P., et al. Structural and dynamical characterization of Hele-Shaw viscous fingering. Philosophical Transactions of the Royal Society A: Mathematical, Physical and Engineering Sciences 2004:1723-1734. https://doi.org/10.1098/rsta.2004.1398

[16] Kang Q., Zhang D., Chen S. Immiscible displacement in a channel: Simulations of fingering in two dimensions. Advances in Water Resources 2004:27(1):13-22. https://doi.org/10.1016/j.advwatres.2003.10.002

[17] Dong B., et al. Lattice Boltzmann simulation of viscous fingering phenomenon of immiscible fluids displacement in a channel. Computers and Fluids 2010:39(5):768-779. https://doi.org/10.1016/j.compfluid.2009.12.005

[18] Bhatnagar P. L., Gross E. P., Krook M. A model for collision processes in gases. I. Small amplitude processes in charged and neutral one-component systems. Physical Review 1954:94(3):511. https://doi.org/10.1103/PhysRev.94.511

[19] He X., Luo L. S. Theory of the lattice Boltzmann method: From the Boltzmann equation to the lattice Boltzmann equation. Physical Review E - Statistical Physics, Plasmas, Fluids, and Related Interdisciplinary Topics 1997:55(6):6811-6820. https://doi.org/10.1103/PhysRevE.56.6811

[20] Qian Y. H., D’Humières D., Lallemand P. Lattice BGK models for Navier-Stokes Equation. Epl 1992:17(6):479-484. https://doi.org/10.1209/0295-5075/17/6/001

[21] Tang G. H., Tao W. Q., He Y. L. Thermal boundary condition for the thermal lattice Boltzmann equation. Physical Review E - Statistical, Nonlinear, and Soft Matter Physics 2005:72(1). https://doi.org/10.1103/PhysRevE.72.016703 
[22] Chen Z., Shu C., Tan D. Three-dimensional simplified and unconditionally stable lattice Boltzmann method for incompressible isothermal and thermal flows. Physics of Fluids 2017:29(5). https://doi.org/10.1063/1.4983339

[23] Chen S., Martínez D., Mei R. On boundary conditions in lattice Boltzmann methods. Physics of Fluids 1996:8(9):25272536. https://doi.org/10.1063/1.869035

[24] Sbragaglia M., Succi S. Analytical calculation of slip flow in lattice Boltzmann models with kinetic boundary conditions. Physics of Fluids 2005:17(9):1-8. https://doi.org/10.1063/1.2044829

[25] Zou Q., He X. On pressure and velocity boundary conditions for the lattice Boltzmann BGK model. Physics of Fluids 1997:9(6):1591-1598. https://doi.org/10.1063/1.869307

[26] Crane Company. 1988. Flow of fluids through valves, fittings, and pipe. Technical Paper No. 410 (TP 410). Springville: Vervante, 1998.

[27] Dhaundiyal A., Tewari, P. Kinetic parameters for the thermal decomposition of forest waste using distributed activation energy model (DAEM). Environmental and Climate Technologies 2017:19(1):15-32. https://doi.org/10.1515/rtuect2017-0002

[28] Dhaundiyal A., et al. Determination of Kinetic Parameters for the Thermal Decomposition of Parthenium hysterophorus. Environmental and Climate Technologies 2018:22(1):5-21. https://doi.org/10.1515/rtuect-2018-0001

[29] Dhaundiyal A., et al. Clayton copula as an alternative perspective of multi-reaction model. Environmental and Climate Technologies 2018:22(1):83-106. https://doi.org/10.2478/rtuect-2018-0006

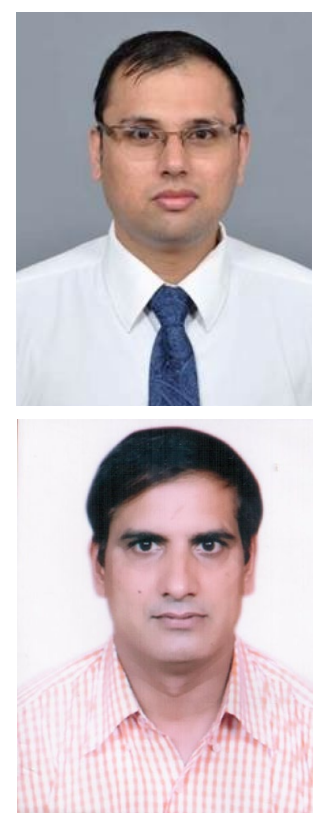

Alok Dhaundiyal. In 2014, he had been awarded the master's degree in Mechanical Engineering (Thermal Engineering) from GBPUAT, Pantnagar, Uttarakhand, India. From 2015 to 2017, he worked as an Assistant Professor in Himgiri Zee University, India. The author's area of expertise is the energy generation from the biomass waste. In $2017, \mathrm{He}$ received the Stipendium Hungaricum award granted by the government of Hungary. The Faculty of Engineering Excellence award has been conferred on him by the University of Strathclyde, Scotland, United Kingdom. He has also received University Grants Commission (from 2017 to 2022) and GATE (from 2012 to 2014) scholarships for his higher studies. Currently, he is the doctoral student (Mechanical) in Szent Istvan University (St. Stephen University), Hungary. He is also a member of the American Chemical Society.

E-mail: alok.dext@hotmail.com

ORCID iD: https://orcid.org/0000-0002-3390-0860

Suraj Bhan Singh is a Professor in the Department of Mathematics, Statistics and Computer Science, G. B. Pant University of Agriculture and Technology, Pantnagar, India. He has around 25 years of teaching and research experience with Undergraduate and Post Graduate students at different Engineering Colleges and University. Prof. Singh is a member of Indian Mathematical Society, Operations Research Society of India, ISST, and National Society for Prevention of Blindness in India and Member of Indian Science Congress Association. He is a regular reviewer of many books and International/National Journals. Prof. Singh has been a member of organizing committees in many international and national conferences and workshops. He is the Editor in Chief of the Journal of Reliability and Statistical Studies. He has authored and co-authored eight books on different courses of Applied/ Engineering Mathematics. He has been conferred with five national awards. He has published his research works at national and international journals of repute. Author's area of research is Reliability Theory.

E-mail: drsurajbsingh@yahoo.com

ORCID iD: https://orcid.org/0000-0003-3557-8139 